\title{
Sprachlos? Erzählen im interkulturellen Kontext
}

\author{
Ein Projekt zur Sprachförderung von Kindern mit \\ Migrationshintergrund
}

Kristin Wardetzky und Christiane Weigel

\begin{abstract}
Zusammenfassung
Der folgende Artikel beschreibt ein Projekt zur Sprachförderung von Kindern mit Migrationshintergrund durch die Praxis des Erzählens. Das Projekt wurde von September 2005 - Juli 2007 in der Anna-Lindh-Grundschule in Berlin Wedding durchgeführt und erhielt 2007 den Sonderpreis der Jury „Kinder zum Olymp“ für „,ein herausragendes Projekt mit ganz besonderer Ausstrahlung, hoher künstlerischer Qualität, innovativer Konzeption und Übertragbarkeit, das deutlich macht, wie Kunst und Kultur Integration im Schulalltag und darüber hinaus nachhaltig befördern werden können“" (Kulturstiftung der Länder, Pressmitteilung vom 23.05.2007). Der Artikel beschreibt die zentrale Projektidee, Erzählen als Mittel zum aktiven Spracherwerb einzusetzen, deren Umsetzung im konkreten Projektsetting und - anhand zahlreicher Beispiele - die im Projektverlauf dokumentierten Erfolge der Interventionen.
\end{abstract}

\section{Die Projektidee}

\subsection{Sprachbabel: Sprachenvielfalt und Sprachlosigkeit in einer Berliner Grundschule}

22 Kinder zwischen 5 und 7 Jahren sitzen als frisch gebackene Schüler und Schülerinnen der Klasse 1e in einem Klassenzimmer der Anna-Lindh-Schule in Berlin Wedding. Mehr als die Hälfte von ihnen stammt aus Elternhäusern nicht-deutscher Herkunft. Sie sprechen zu Hause türkisch, arabisch, bosnisch, russisch, polnisch, englisch, französisch, ... oft auch eine eigentümliche Mischung aus verschiedenen Sprachen, denn selbst die Muttersprache wird häufig nur lückenhaft beherrscht. Die Einschulung bedeutet für einige der Kinder einen erstmaligen Kontakt mit hochdeutscher Sprache. 80 \% der Schüler und Schülerinnen wurde zu Schulbeginn eine schlechte bis ungenügende Beherrschung der deutschen Sprache bescheinigt. ${ }^{1}$

\footnotetext{
${ }^{1}$ Ermittelt im Juni 2005 durch den Sprachstandstest ,Bärenstark.'
} 
Hochdeutsch spricht allerdings auch in der Schule nur die Lehrerin. Untereinander verständigen sich die Kinder in dem nicht nur für den Wedding typischen Soziolekt der deutschen Sprache: kurze, grammatikalisch einfache, häufig unvollständige Sätze mit einem stark begrenzten Vokabular, häufig durchwoben von türkischen, englischen, arabischen Begriffen. Es ist die Kunst, mit möglichst wenig Wörtern möglichst viel auszudrücken. In vielen Situationen sind die Kinder aber auch schlicht 'sprachlos', nicht dazu in der Lage, verbal angemessen auf eine Situation zu reagieren. Dies führt - vermutlich - nicht selten zu körperlichen Ersatzhandlungen, die diese Hilflosigkeit kompensieren.

Viele der neuen ABC-Schützen sind im Schulalltag rasch überfordert. Die sprachliche Orientierungslosigkeit ist dabei nur Symptom einer kulturellen und sozialen Fremdheit, mit der viele von ihnen im System Schule konfrontiert sind.

S. ist eines der 176 an unserem Projekt beteiligten Kinder - ein blasses dünnes Mädchen, das jeden Morgen zu spät kommt, weil ihre Mutter sie nicht weckt und sie sich morgens selbst versorgen muss. Auch an ihrem Geburtstag steht niemand mit ihr auf und überreicht ihr Geschenke. S. hat keinen Tuschkasten und keine Turnsachen, obwohl die Lehrerin schon viele Mitteilungen an ihre Mutter geschrieben hat. Frühstücksmilch und Mittagessen sind für sie nicht bezahlt, aber S. hat sich Strategien erarbeitet, um auf halblegalem Wege dennoch an das für sie Nötigste zu kommen. Und das ist ihr auch lieber als die Scham, die sie empfindet, wenn eine Lehrerin auf ihre Mängel aufmerksam wird. Bei ihr zu Hause gibt es Geschichten höchstens aus dem Fernsehen. Zweimal in ihrem Leben war sie schon in einem Kino, mit einer Frau vom Jugendamt, die sie manchmal besucht. „Die ist auch in deutsch“, sagt S. „Aber ich bin nicht Deutschland. Ich bin Bosnien."

\subsection{Erzählen als Mittel zum aktiven Spracherwerb}

Das Projekt geht auf Erfahrungen zurück, welche Sabine Kolbe, Kerstin Otto und Marietta Rohrer-Ipekkaya während ihrer mehrjährigen Berufspraxis als professionelle Erzählerinnen mit Kindern machten. Wurden von ihnen Märchen und Mythen erzählt (nicht zu verwechseln mit dem Vorlesen!), dann war zu erleben, wie Kinder höchst konzentriert erstaunlich lange zuhören - vor allem Kinder mit Migrationshintergrund, die in der deutschen Sprache nicht heimisch sind, sowie hyperaktive und sogenannte, verhaltensauffällige' Kinder und Kinder aus bildungsfernen Elternhäusern - und dies allein über die Macht des gesprochenen Wortes.

Zwei Jahre lang kamen die drei genannten Erzählerinnen (als solche ausgebildet an der Universität der Künste Berlin) in den regulären Deutschunterricht der zwei 1. und zwei 2. Klassen der Anna-Lindh-Grundschule in Berlin-Wedding und erzählten anfangs zweimal, nach einem halben Jahr einmal pro Woche internationale Märchen.

Das Projekt wurde initiiert und betreut von Kristin Wardetzky, Universität der Künste Berlin, Institut für Theaterpädagogik, und dokumentarisch begleitet von Felix Strasser und Christiane Weigel, Absolventen des Instituts. 


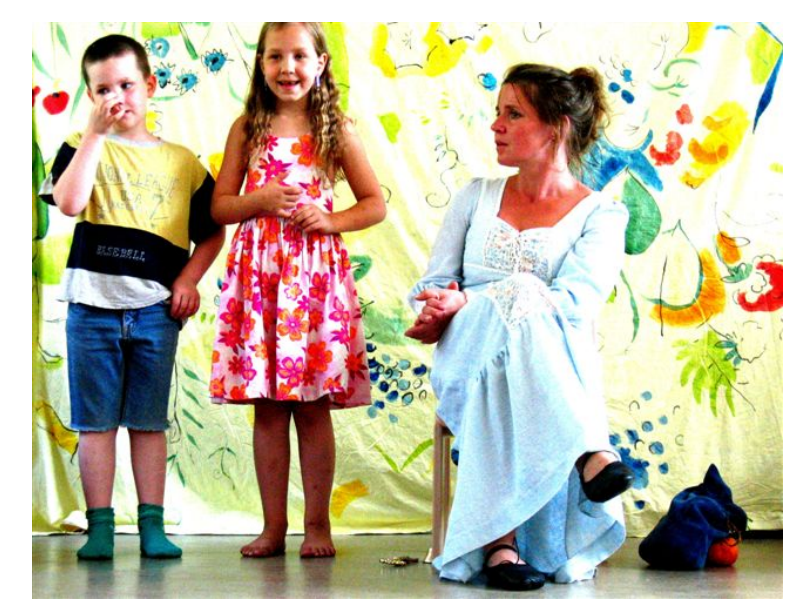

Abbildung 1: Erzählerin Kerstin Otto mit Kindern

Die Finanzierung dieses Drittmittelprojektes erfolgte über die Deutsche Bank Stiftung, die Volkswagen AG, die LBS Nord und Innerwheel Berlin-Mitte.

Zum Projektverlauf: Zunächst erzählten die Erzählerinnen kürzere Märchen von 7-10 Minuten und steigerten dann die Länge und Komplexität der Geschichten. Am Ende wurden Geschichten von einer Länge bis zu 45 Minuten erzählt. An den jeweiligen Erzählvorgang schlossen sich theaterpädagogische Sequenzen an (Bewegungs- und Sprachspiele, szenische Improvisationen etc.). Nach ca. 6 Monaten wurden die Kinder angehalten, eigene Geschichten zu erfinden und vor der Klasse zu präsentieren. Rezeptives und produktives Erzählen kamen in ein ausbalanciertes Verhältnis.

Zur dokumentarischen Begleitung: Felix Strasser und Christiane Weigel protokollierten nach vorgegebenen Kriterien sprachliche und nonverbale Reaktionen der Kinder in den Erzählstunden. Christiane Weigel führte im 2. Projektjahr Einzelinterviews mit den beteiligten Kindern durch, in denen diese die gehörten Märchen nacherzählten oder eigene Geschichten erfanden.

Die Praxis des Erzählens war anfangs den Schülern, auch denen ohne Migrationshintergrund, weitgehend unbekannt. Das Fernsehen als omnipräsenter Familienunterhalter bestimmt zu einem Großteil ihren Familienalltag. Märchenoder Kinderbücher sind in den Haushalten kaum oder gar nicht vorhanden. So antworteten Kinder auf die Frage, ob ihnen zu Hause Geschichten erzählt würden:

„Meine Mama und Papa macht das nicht. Meine Mama geht an Computer und spielt so. Sie geht ans Telefon: 660060. Dann ist Carsten dran, mein Papa und erzählt von dem Spiel. Da kann man kämpfen. Finde ich langweilig. Meine Mama erzählt nie Geschichten.“

„Nein. Aber ich war krank, da hab' ich ein Buch von Doktor gekriegt. Da les' ich oft drin. Da steht von Hals. Dass man immer trinken muss und Hand vor Mund.“

„Das macht meine Mutter nicht. Weil sie immer telefoniert, am Computer 
sitzt, und dann ist es dunkel, und dann sind wir im Bett. Aber ich erzähl meine Mama jetzt.“

Vor diesem Hintergrund bestimmten wir das Projekt als ein dezidiert sprachgeleitetes, in dessen Mittelpunkt das Eindringen in die deutsche Sprache und der Umgang mit ihr stand. Dies scheint für den Unterricht im allgemeinen als eine Selbstverständlichkeit. Unser Projekt unterscheidet sich insofern von anderen didaktischen Verfahren, als es zum einen nicht am (defizitären) Sprachniveau der in das Projekt einbezogenen Schüler ansetzte, sondern eine poetische, vom Alltagsidiom unterschiedene Sprache behauptete. Die Erzählerinnen boten das gesamte Repertoire ausgefeilten hochdeutschen Erzählvokabulars auf.

Zum anderen vermittelte es Sprache nicht abstrakt-begrifflich / grammatikalisch / orthografisch, sondern in aufrüttelnden, spannungsgeladenen Geschichten. Die Kinder erlebten in diesem Projekt Sprache primär in ihrer emotional sinnlichen Qualität, und eben dies erwies sich als wirkungsvolles Instrument der,Verführung' zum Zuhören und zum kreativen Sprachgebrauch. Der Spracherwerb vollzog sich hier in einem Prozess mit hoher emotionaler Anteilnahme. Dies mag ausschlaggebend für die Effizienz des Verfahrens gewesen sein.

Wie machten sich die Erzählerinnen den Kindern, die z.T. mit erheblichen Schwierigkeiten in der deutschen Sprache zu kämpfen hatten, verständlich? Die Antwort scheint simpel: durch Erzählen! Anders als beim Lesen hält das Erzählen eine Verständigungsebene jenseits des ,reinen' Wortes bereit: Beim Erzählen erscheinen die Wörter eingebettet in ein dichtes Netz von sinnlich Wahrnehmbarem, das dem Zuhörer kaum bewusst wird, aber dennoch für den Aufbau des Verständnisses einer Geschichte konstitutiv ist: Die Vibration der Stimme, ihre Tonhöhe, die Modulation des Tempos, der Lautstärke, der Satzmelodie, dazu die vielgestaltigen Möglichkeiten der ,beredten“ Mimik, der Gestik, der Körperhaltung - all das erzählt mitunter mehr und anderes als Worte sagen. Da diese Mittel beim Erzählen stärker zum Tragen kommen als beim Vorlesen, ist es im Unterricht letzterem noch immer überlegen, auch wenn das Vorlesen eine nicht zu unterschätzende Möglichkeit der literarischen Kommunikation darstellt. Der Erzähler aktiviert - spontan oder wohlkalkuliert - alle Möglichkeiten seines expressiven Repertoires und macht sich damit verständlich, auch wenn einzelne Worte unverständlich bleiben. Dies macht die Bedeutung des Erzählens in der multikulturellen Schulpraxis aus: Es hält eine Verständigungsebene bereit, der auch nicht-muttersprachliche Kinder zu folgen vermögen, und zwar genussvoll und konzentriert.

Im Erzählen kommt ein Weiteres hinzu, das beim Vorlesen nur eingeschränkt seine Wirkung entfalten kann: der Blickkontakt und die Möglichkeit, sensibel und spontan auf situative Reaktionen der Kinder einzugehen. Über die Augen und zunächst nur über sie - wird eine Primärkonstante gelingenden Erzählens garantiert: der Kontakt. Mit den Augen holt der Erzähler die Zuhörer zu sich, über die Augen lässt er sie an der von ihm geschaffenen Welt des Abenteuers teilhaben, oder besser noch: gemeinsam mit den Zuhörern lässt er diese Welt entstehen. 
Umgekehrt bleiben dem Erzähler über den Blickkontakt die Reaktionen der Zuhörer nicht verborgen. Er kann sie aufnehmen, auf sie ganz unmittelbar reagieren, sie zum Bestandteil seiner Erzählung machen, da er frei ist im Text, ihn nicht, wie beim Vorlesen, unter- oder abbrechen muss, um auf die Zuhörer direkt einzugehen. Solche situative Flexibilität setzt allerdings ein hohes Maß an Professionalität, also umfangreiche Erfahrungen im Erzählen voraus.

\section{Ergebnisse des Projektes}

\subsection{Zuhören und Repetieren als Wege zum Spracherwerb}

Nach zwei Jahren Projektzeitraum, in dem die Kinder das wöchentliche Erzählen genossen, war das Lehrpersonal verblüfft über die gewachsene Fähigkeit der Kinder, zuzuhören statt lediglich hinzuhören. „Hinhören“, so eine der am Projekt beteiligten Lehrerinnen, „das tun sie. Das kennen sie von zu Hause. ,Mach das..., lass jenes..., hör auf...': Hinhören und Abschalten, das ist der Alltag in den Familien. Aber jetzt kann man richtig sehen, wie sie beim Zuhören anfangen zu denken!" Zuhören ist eine Grundvoraussetzung menschlicher Kommunikation und hiermit unabdingbar für schulisches Lernen. Auch diese ist über Erzählen vermittelbar. Eine andere Lehrerin:

Und dann ist es natürlich fantastisch, die Kinder zu beobachten, wie die da mit offenem Mund sitzen und mit glühenden Augen und mitgehen. Das ist schon sehr, sehr schön. Wenn manche Kinder mal schlecht drauf sind, und wenn die dann total überdreht sind, wie die Erzählerinnen das einfach schaffen durch ihre künstlerische Art, eben diese Kinder zur Ruhe zu bringen. Dass sie sich so zurücknehmen und innere Ruhe finden.

Die das Projekt steuernde Vorannahme, dass sprachlich vermittelte Märchenrezeption die kindliche Sprachentwicklung entscheidend beeinflussen kann, wurde durch vielfältige verbale Rückmeldungen, Einwürfe, Ergänzungen der Kinder bestätigt. Zuhören erwies sich als aktiver Spracherwerb; Sinnkonstitution und Sinnverständnis erfolgte beim Zuhörern durch das Ineinander von Erkennen, Empfinden und Werten. Die Erkenntnis des semantischen Gehalts einzelner Wörter wird durch deren Einbettung in ein affektiv erlebtes Geschehen erleichtert. Emotionen befördern intellektuelles Verstehen. Zwei Beispiele:

Kerstin Otto gebrauchte in der Geschichte,Vom dummen Fuchs' verschiedene Ausdrucksweisen um zu beschreiben, wie der schlaue Fuchs den dummen Wolf an der Nase herumführt. Die Kinder, welche für derartige Vorgänge bisher nur die Formulierung ,jemanden verarschen' oder ,jemanden austrixcksen' kannten, lernten im Kontext der Erzählung ohne ausdrückliche Erklärung, was es bedeutet ,jemanden an der Nase herumzuführen' oder ,jemanden aus etwas einen Strick zu drehen'.

In einer anderen Stunde verwendete sie mehrfach den Spruch: „Auf des Hecht's Geheiß, so wie ich's wünsche, so sei's.“ Als sie im Anschluss fragte, ob die Kinder den Sinn des Spruches erklären könnten, gelang dies einem Mädchen 
umstandslos, und auch die anderen schienen ,Geheiß' sinngemäß verstanden zu haben.

Mitunter ergaben sich kuriose Missverständnisse. So identifizierten die Kinder einen ,Müller' als den ,Mann, der den Müll runterbringt'; Kohle übersetzten sie sich mit ,Cola' und waren bass erstaunt, dass man damit einen Ofen heizen kann. Die ,Spree' wurde für sie zu ,Spray', die Donau zu ,Döner'. In einigen Fällen lösten unbekannte Wörter auch ein direktes Gespräch aus, in dem ihre Bedeutung diskutiert und geklärt wurde. So führte eine Geschichte über Leiden zu der Frage eines Kindes: „Was ist denn Leiden noch mal?"Ein Junge erwiderte: „Wenn man sich so quält.“ Worauf ein anderes Kind zu bedenken gab: „Aber leiden heißt doch mögen!"

Unbekannte Schlüsselbegriffe, die für das Verständnis der Geschichte zentrale Bedeutung hatten, wurden meist vor Beginn der Geschichte erläutert. Die wiederholte Begegnung mit den neuen Begriffen im Verlauf der Geschichte und ihre Verwendung in unterschiedlichen Zusammenhängen festigte sie im Gedächtnis der Kinder. Was ist eigentlich ,Hölle', was bedeutet,Argwohn', wohin geht der Prinz, wenn er in eine ,Kammer' geht, welche ,Kunde' wird verbreitet, was ist ,segnen', was ist eine Ziege? Das sind einige der Fragen, die in den Erzählstunden auftauchten, aber in den seltensten Fällen direkt erklärt wurden wie im Deutschunterricht. Am Ende der Geschichte hatten die Kinder in der Regel ohne explizite Erklärung Verständnis für die Bedeutung und Verwendung des neuen Wortes entwickelt. In den besten Fällen benutzten sie es selbst, wenn sie die Geschichte nacherzählten.

Vielfach verwendeten die Erzählerinnen repetitive Formen des Spracherwerbs. Sie ließen wiederkehrende Verse und Reime von den Kindern mitsprechen, nachsprechen, voraus sprechen, und dies führte (fast) immer dazu, dass die Kinder die mitgesprochenen Ausdrücke im Anschluss selbstständig wiederholen und erklären konnten.

Auffällige sprachliche Defizite waren insbesondere bei der Bildung von Zeitformen zu beobachten. Die Bildung des einfachen Präteritums wurde von den Kindern in der Regel vermieden. Die Märchen konfrontierten sie mit komplexen Zeitformen und vor allem mit der Schwierigkeit in der Beugung starker Verben. Die für das Märchen (beinahe) obligatorische Zeitform ist das Präteritum. Im Verlauf des Projektes begannen Kinder in ihren Nacherzählungen und Erfindungen zunehmend, mit dem Präteritum zu experimentieren. Aus ,danach geht er in den Wald' wurde ,er gang in den Wald', dann ,er ging in den Wald'.

Vielfach schien den Kindern beim Versuch, die gehörten Geschichten nachzuerzählen, die Differenz zwischen dem sprachlichen Ausdruck der Erzählerinnen und ihren sprachlichen Möglichkeiten bewusst zu werden. Ihre Nacherzählungen dokumentieren den deutlichen Versuch, der Plastizität der Schilderung durch die Erzählerin nahe kommen zu wollen. Ein Beispiel:

I. ist einer der Jungen, der beim Erzählen sichtlich unter seinen sprachlichen Barrieren leidet. Er erzählt sehr langsam, denkt zwischendurch lange nach und sucht in der Erinnerung nach den richtigen Worten. Er wirkt geradezu 
zerknirscht, wenn er an einer Stelle ins Stocken gerät. Es quält ihn, Dinge nicht erzählen zu können, die eigentlich in seiner Vorstellung vorhanden sind: „Ich hab noch mehr verstanden. Ich kann bloß die Sprache nicht so gut." Dennoch bemüht er sich innerhalb seiner Möglichkeiten, Lösungen zu finden. In der von ihm erzählten Geschichte gibt es eine Verheißung bei der Geburt des Jungen für sein 14. Lebensjahr. I. probiert sich im Konjunktiv: „Der wird, wenn er 14 wäre, dann würde er die Prinzessin heiraten." An anderer Stelle versucht er, das Präteritum zu bilden und arbeitet mit Wiederholung, um die von der Erzählerin sehr intensiv vermittelte lange Dauer der Reise wieder zu geben. „Und er gang und gang und gang bis zu einem Baum."

Deutliche Wirkungen zeigte die Arbeit mit Wortspielen verschiedenster Art. So wurde z. B. die von Franz Fühmann übernommene Geschichte vom Reh am See, welches den Klee im Schnee aß, also das ,Seeschneekleereh', für Sabine Kolbe zum Ausgangspunkt, um mit den Kindern eigene Reimgeschichten zu erfinden. Das Reimen machte den Kindern nicht nur viel Spaß, sondern erleichterte es ihnen auch, neue Wörter auszusprechen und sich einzuprägen.

In gleicher Weise lustvoll erlebten die Kinder den Umgang mit Kettenmärchen. In diesem Genre wird kurz ein Anfangskonflikt etabliert, dann folgen sukzessiv nacheinander kurze Episoden von geringer Variabilität. Jeweils wird das Ganze von Anfang an wiederholt und damit um je ein neues Kettenglied verlängert. Kinder haben dieses Reihungsprinzip schnell durchschaut und sprechen die Einzelepisoden mit, schneller und schneller, am Ende beinahe außer Atem. Da sich diese Märchen bei den Kindern besonderer Beliebtheit erfreuten und ihrem Drang nach Partizipation entgegenkamen, wurden sie vielfach am Ende einer Erzählstunde wiederholt. Diese Kettenmärchen weisen eine starke Rhythmisierung auf und evozieren körperliche Begleitbewegungen. Durch dieses Ineinander von sprachlichem und motorischem Memorieren wird die Verankerung im Gedächtnis nachhaltig unterstützt. Auch hier lernten die Kinder Begriffe, Formulierungen und Wendungen kennen, die in der Umgangssprache kaum oder keine Verwendung finden, die ihnen jedoch im weiteren Verlauf zum aktiven Gebrauch zur Verfügung standen.

\subsection{Kostbare Begegnung mit der eigenen Sprache}

Fragte man die Kinder: „Wie sprechen wir im Unterricht?“, so lautete die eingeübte chorische Antwort: „Deutsch!“ Abgesehen von der in der Regel etwas größeren Gemeinschaft türkischer und arabischer Kinder waren die meisten Kinder mit ihrer Muttersprache in der Schule auf einsamem Posten. Gezwungen, sich in der meist nur mangelhaft erlernten deutschen Sprache zu verständigen, litten viele Kinder darunter, in der Schule ihrer ursprünglichen sprachlichen Identität beraubt zu sein.

M. und S. kommen zu Beginn des Schuljahres gemeinsam neu in die Klasse 2. Beide stammen aus dem ehemaligen Jugoslawien und sind in diesem Sommer mit ihrer Familie nach Deutschland gekommen. An ihrem ersten Schultag in der Anna-Lindh-Grundschule sprechen sie kein Wort Deutsch. Während die 
Kinder sich in den Pausen spielerisch auch ohne Sprache näher kommen, stehen die Lehrer und die neuen Schüler gleichermaßen hilflos vor dem Problem der Kommunikation mit zwei komplett ,sprachlosen' Menschen.

Ein halbes Jahr später hat M. es geschafft. Er hat die Grundzüge der deutschen Sprache erlernt und ist zu einem guten und sehr beliebten Schüler der Klasse geworden. S. dagegen hat das Sprechen fast gänzlich eingestellt. Mit seinen Mitschülern kommuniziert er über seinen Körper, eine lebhafte Mimik und Gestik; den Lehrern gegenüber verweigert er das Sprechen. Im Unterricht fällt er durch ein extremes und besorgniserregendes Störverhalten auf, was nicht weiter verwunderlich erscheint für ein Kind, dass täglich bis zu 6 Stunden Unterricht hinter sich bringt ohne etwas von dem zu verstehen, was dort verhandelt wird. S. hat Strategien entwickelt, sich die Zeit anders zu vertreiben. Das Klassenziel wird er allerdings nicht erreichen, am Schuljahresende wiederholt er das Schuljahr.

Die Erzählerinnen bemühten sich zunehmend, die kulturelle und sprachliche Vielfalt der Kinder aufzugreifen und in das Erzählen zu integrieren. Sie suchten solche Märchen aus, die aus den Herkunftsländern der Familien der Kinder stammten. Wenn möglich, flochten sie bestimmte wiederkehrende Wendungen (Verse, Eingangs- und Schlussformeln) in der jeweiligen Nationalsprache ein. Dadurch entstanden wirkliche Glücksmomente für Kinder, wenn sie plötzlich in ihrer eigenen Sprache angesprochen wurden, ihre Muttersprache erkannten und einzelne Wendungen mitsprechen konnten. Dieses Glück resultierte sicher maßgeblich aus der Erfahrung der Kinder, dass ihre Muttersprache in der Schule unbekannt und unerwünscht war.

„Ist das von Russland? Ich bin Russland!“ rief der siebenjährige E., als in einer Geschichte die ,Baba Jaga' auftauchte. Endlich einmal war er der Wissende und Eingeweihte, stand seine Sprache und seine Kultur im Mittelpunkt des Geschehens.

Es wurde deutlich, dass die Erfahrung der sprachlichen Zurücksetzung nicht unerheblich dazu beitrug, dass Kinder das Zuhören und Lernen verweigern. So gab es einen ebenfalls russischen Jungen in einer Klasse, der monatelang die Erzählstunden massiv störte und die gesamte Klasse mit seiner Verweigerungshaltung in Aufruhr brachte. Als H. wieder einmal eine Erzählstunde sabotierte, forderte die Erzählerin ihn auf, die von ihr erzählte russische Geschichte in seiner Sprache wiederzugeben. Nach anfänglicher Hemmung geriet der Junge mehr und mehr in konzentriertes Erzählen, während die restliche Klasse wahrscheinlich zum ersten Mal und höchst erstaunt ihren Mitschüler flüssig und wortgewaltig reden hörte. Als Folge wollten nicht nur alle anderen Kinder die Geschichte ebenfalls in ihrer Muttersprache erzählen, sondern H. war in den kommenden Stunden wie ausgewechselt, sein Interesse am Erzählen geweckt.

Gerade Kinder, die durch einen kurz zurückliegenden Wechsel von Heimat und Sprache noch verstört und sprachlich höchst hilflos dem Unterricht beiwohnten, konnten in Erzählstunden mit Geschichten und sprachlichen Wendungen aus ihrem Heimatland berührt und in ihrer Aufmerksamkeit geweckt werden. 


\subsection{Empathie - wie Kinder als Zuhörer mitfühlen}

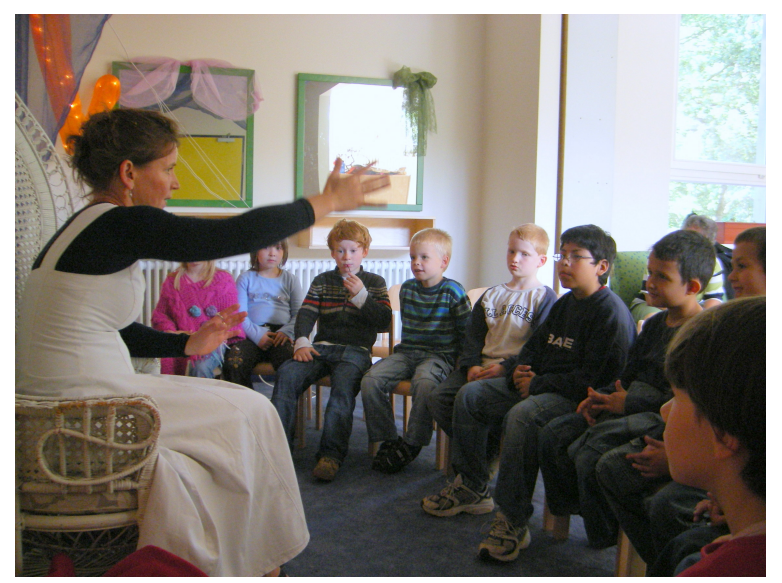

Abbildung 2: Kerstin Otto erzählt

Spontane verbale Äußerungen der Kinder, ihre Mimik oder Gesten, in denen sich aufgestaute Spannungen entluden, ließen die innerliche Beteiligung der Kinder am erzählten Geschehen sichtbar werden. Einzelne Kinder zeigten von Anfang an starke körperliche Reaktionen auf das Erzählte, und der Großteil entwickelte im Laufe des Projektes einen zunehmend mimischen und körperlichen Ausdruck von Empathie. Je stärker die Erzählerinnen in Tonfall, Gestik und Mimik Figuren lebendig werden ließen, desto auffälliger wurden diese von den Kindern aufgenommen und gespiegelt. Kinder ahmten die tiefe Stimme der Erzählerin als Sultan nach, schwenkten selbst als Kämpfer ein imaginäres Schwert in der Luft, zeigten in ihren Gesichtern Angst, Freude, Schmerz oder Zorn der Protagonisten. Die Entwicklung hin zu einer stärkeren verbalen Erzählbeteiligung der Kinder im zweiten Schuljahr führte außerdem dazu, dass die Kinder sich immer stärker in das Erzählen einmischten, Erzähltes kommentierten und sogar versuchten, unbekannte Geschichten mit der Erzählerin mitzusprechen. Ihre Kommentare machten deutlich, dass sie die Inhalte emotional mit durchlebten und sich in starker Weise mit den Protagonisten der Märchen identifizierten. Einige Beispiele:

Erzählerin: „Zum letzten Mal: Wirf meine Frau und meinen Sohn ins Feuer!“ Ein Kind reißt die Arme hoch und ruft voller Angst: „Nein!“

Erzählerin: „Der Wirt tauscht also heimlich - wie schon das Tüchlein den Goldesel gegen einen gewöhnlichen Esel aus." Spontane Zurufe der Kinder: „Mann eh, voll gemein.“ „Er will immer alles klauen.“ „Lügner!“ „Verräter!“

Erzählerin: „Als der Teufel wieder eingeschlafen war, riss des Teufels Großmutter ein drittes Haar vom Kopf des Teufels“. Ein Kind: „Oh nein! Nicht schon wieder! Sonst tötet er sie!“

Die von den Erzählerinnen ausgewählten Märchen scheuten nicht vor der Darstellung von Grausamkeiten zurück. Wie reagieren Kinder darauf, deren Enkulturation zu einem Großteil durch Fernseh-, Video- und Computerkonsum 
bestimmt ist?

Die Schwierigkeiten der Kinder lagen, und das war uns eingangs nicht bewusst, nicht nur im Bereich der sprachlichen Defizite, sondern insbesondere im Bereich der Phantasie. Bei einem erheblichen Teil der Kinder war die Imaginationsfähigkeit blockiert. In oft erschreckender Weise besetzten auch bei den Erstklässlern Sex and Crime diesen Platz. D.h., die Landkarte der Phantasie dieser Kinder ist beschrieben; eingezeichnet aber sind medial vorgefertigte Bilder.

Zu Beginn des Projektes waren besonders die selbst erfundenen Geschichten der Kinder von Gewalt geprägt. So bekamen die Kinder in einer Stunde die Aufgabe, Lügengeschichten zu erzählen. Diese Geschichten handelten allesamt von Kämpfen, Töten, explodierenden Bomben. Anwesende Kinder kamen in den Schilderungen der Mitschüler zuhauf zu Tode. S.: „Der hat eine Granate geworfen. Ist explodiert. Valentino (eines der anwesenden Kinder) hat gestorben und seine Beine waren Salami. Sind sie zu Gott und der hat ihm noch eine letzte Chance gegeben. Sind sie im Haus gegangen. Im Haus war eine Bombe. Das Haus explodiert. "Die Kinder lachten sich bei jeder Explosion kaputt und wirkten geradezu high, wodurch der jeweilige Erzähler weiter angefeuert wurde. Die Erzählerin fragte die Kinder anschließend, ob sie zu Hause auch Bombenexplosionen spielten. „Ja.“ antwortete M., „im Computer gibt es den Torpedo. Da kann man eine ganze Titanic zerstören.“ Erzählerin: „Und das macht euch Spaß?" Ein einstimmiges euphorisches „Jaaaa!“ als Antwort.

Allmählich gewannen auch die Bilder der Märchen Raum in ihren Köpfen. Ein erstaunlicher Befund stellte sich ein: Kinder, die beim Erzählen selbsterfundener Gewaltsensationen, Blut- und Racheorgien in ein ungezügeltes, aggressives Lachen ausbrachen, reagierten beim Anhören der im Märchen erwähnten Grausamkeiten gänzlich anders: Sie blieben gelassen, konzentriert. Keine Lacher, kaum körperliche Reaktionen. D.h. sie wehrten offensichtlich nicht durch frenetisches Gelächter eine emotionale Irritation oder gar Überwältigung ab. Wie derartige psychische Prozesse tatsächlich ablaufen, bildet ein Forschungsdesiderat. Unsere Beobachtungen legen die Annahme nahe, dass die jeweilige Rezeption auf zwei unterschiedlichen, voneinander getrennten Wahrnehmungs- und Verarbeitungsebenen stattfindet, und dass es zwischen beiden kaum Interdependenzen zu geben scheint. Die symbolische Darstellung scheint einer Art Schutzfunktion gleichzukommen. Vergegenwärtigt, in Bilder übersetzt, wird vermutlich nur, was psychisch zu bewältigen ist. Das Symbol markiert die Schwelle zwischen Realität und Fiktion. Die Verlebendigung des Fiktionalen lässt sich steuern; seine Macht kontrollieren.

\subsection{Fallbeispiele}

Im Rückblick macht das Projekt auf eine Reihe von Grundproblemen schulischen Lernens aufmerksam. Eines davon ist die Einbindung von Kindern mit einem problematischen sozialen Hintergrund, die im normalen Unterrichtstalltag durch extreme Lernschwächen, ein hohes Aggressionspotential oder Konzen- 
trationsschwächen wie das ADS-Syndrom auffallen. Sie reagieren bekanntlich besonders sensibel auf Leistungsdruck, ziehen sich aufgrund ständiger Misserfolge entweder zurück oder reagieren mit unkontrollierten emotionalen Ausbrüchen. Vom Lehrpersonal, Mitschülern und/oder Eltern werden sie häufig als ,Problemkinder' wahrgenommen. Eben diese Kinder konnten vom Erzählprojekt in besonderer Weise profitieren.

Drei Beispiele:

H. - ein Junge, der mit gerade acht Jahren bereits zum zweiten Mal wegen massiver körperlicher Handgreiflichkeiten auf dem Schulhof einer Vorladung zur Polizei nachkommen musste, ist es im Unterricht unmöglich, mehrere Minuten still zu sitzen und sich auf eine Sache zu konzentrieren. „Aber bei diesem Erzählprojekt, da ist er so verzaubert, da findet der so seine Insel und entspannt", stellt die Lehrerin fest. In den Erzählstunden ist H. sehr ruhig, verbale Äußerungen gibt es von ihm kaum. Insbesondere bei den Kämpfen der Helden gegen Drachen und andere Ungeheuer ist er jedoch sichtlich körperlich und geistig involviert, und manchmal scheint es, als würde er einen Teil seiner aggressiven Energien abbauen, wenn er sich in den Drachen oder den Helden verwandelt. Im Interview sagt $\mathrm{H}$., er schäme sich, vor der ganzen Klasse Geschichten zu erzählen, beginnt jedoch in der etwas intimeren Situation des Interviews eine Geschichte nach der anderen zu erzählen. Dabei benutzt er erkennbar Elemente aus vergangenen Erzählstunden. Seine Geschichten handeln in erschütternder Weise von Verlassenwerden, Verrat, Verfolgung und Tod. Ein Ausschnitt: Es war einmal ein Wald. Da war nur ein Wildschwein. Nur eins, sonst gar keiner. Das ist gerannt, nach Hause. Da war keiner. „Wo ist Mama?" hat der so geredet, obwohl da keiner war. Nur so geredet. „Mama!“ gerufen, so laut er konnte. Mutter ist gekommen. „Wo warst du? Ich hab dich gesucht.“ Sagt die Mama: „Ich war die ganze Zeit neben dir.“ „Aber ich hab links und rechts geguckt. Da warst du nicht.“ „Dann war ich über dir.“ „Hab ich auch geguckt.“ „Dann war ich zu Hause.“ Sie hat gelogen. Das war auch nicht seine Mutter. Sie hatte eine Maske und so einen Anzug. Sie waren alle tot. Das hat das Wildschwein nicht gewusst.

M. aus Serbien kommt im zweiten Schuljahr neu in die Klasse. Bei seiner Ankunft spricht er kein Wort deutsch. Im Laufe des Schuljahres entwickelt der Junge starke Auffälligkeiten. Er spricht nicht, scheint auch wenig zu verstehen, schaut keinem Erwachsenen in die Augen, verschließt sich immer mehr und entwickelt diverse Strategien, um fünf Stunden sprachlose Langeweile in der Schule durchzustehen. Meistens wird er schon vor der Erzählstunde von der Klassenlehrerin aussortiert und nach hinten gesetzt, weil er im Laufe des Tages eine Liste von Vergehen angesammelt hat. Die Erzählerinnen sind nicht bereit, seine innere Abwesenheit zu akzeptieren und bemühen sich stark um M. Er wird in Geschichten einbezogen, einzelne Passagen werden für ihn übersetzt. Diese explizite Zuwendung ist außerhalb der Märchenstunden nicht aufrecht zu erhalten, sie scheint den Jungen aber sichtlich zu verwandeln. In den nächsten Stunden sitzt M. stets in der ersten Reihe und schaut mit großen erwartungsvollen Augen zur Erzählerin. Hier wird seine Sprachlosigkeit ein 
Stück weit aufgehoben und das scheint ihm sehr gut zu tun.

W. erzählt gern und viel. Allerdings kostet es Zeit und Mühe, ihren Worten in stark polnischem Akzent zu folgen - oft fehlen ihr die Worte, und sie gerät ins Stocken. Im Unterricht wird ihr darum häufig von der Lehrerin das Wort abgeschnitten, auch die Mitschüler meiden den Kontakt zu ihr. Im Interview genießt W. die ungeteilte Aufmerksamkeit und erzählt das komplexe Märchen aus der Erzählstunde in beeindruckender Genauigkeit und Detailtreue nach. Anschließend muss ich (C.W.) ihr das von ihr Erzählte vorlesen, und sie ist vollkommen überrascht und beeindruckt, dass sie allein diese ganze Geschichte erzählt hat. Das hat sie sich nicht zugetraut; das wird ihr auch im Deutschunterricht von niemandem zugetraut.

D. hat massive Sprechprobleme. Er stottert und braucht viel Zeit, um eine Geschichte nachzuerzählen, Zeit, die ihm seine Klassenkameraden vermutlich selten einräumen und die im Unterricht häufig nicht vorhanden ist. Er genießt es sichtlich, im einstündigen Interview in Ruhe um Ausdruck für seine Gedanken ringen zu können. Doch immer wieder neigt er auch zum Aufgeben, wenn die Worte nicht heraus kommen wollen und kritisiert sich selbst. Sein Stolz ist groß als ich (C.W.) ihm zeige, wie viele Seiten er in meinem Buch mit seiner Erzählung gefüllt hat. Er kann die Geschichte gut und zusammenhängend erzählen, stellt sich vieles beim Erzählen bildlich vor und freut sich an diesen Vorstellungen: „Dann kam die wunderschöne Prinzessin. Wie heißt die? Nur Prinzessin? Prinzessin Wunderschön könnte die doch heißen." Als ich ihn frage, ob er nicht auch mal den anderen Kindern eine Geschichte erzählen wolle, antwortet er sofort: „Nein. Ich stotter doch.“ Im Juni 2007, nach zwei weiteren Interviews erzählt D. dann tatsächlich zum ersten Mal vor der Klasse eine kleine Geschichte. Von der Erzählerin unterstützt, wird dies ein großes Erfolgserlebnis für ihn.

\subsection{Entwicklung von Erzählkompetenz}

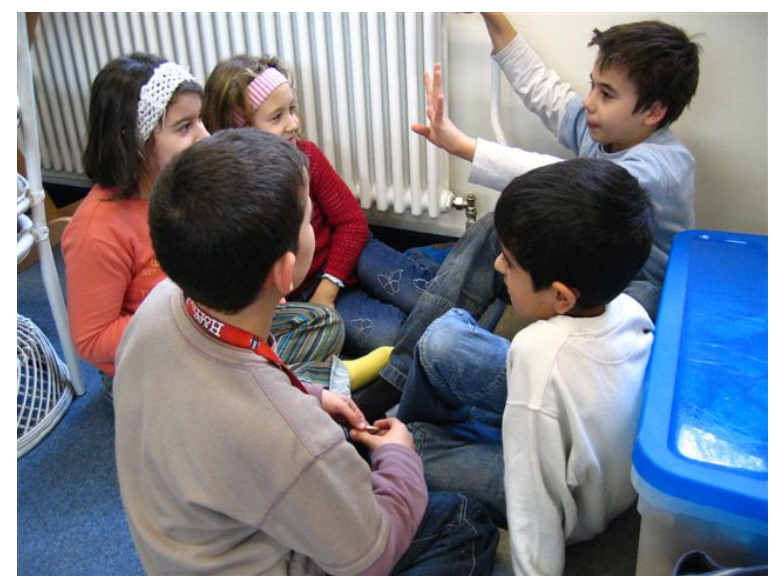

Abbildung 3: Erzählen in Kleingruppen

Ab dem zweiten Projektjahr (Schuljahr 2006/2007) verlagerten sich die 
Proportionen innerhalb des Projektverlaufs zugunsten des Erzählens der Kinder. Dabei nutzten die Erzählerinnen verschiedene Verfahren als Impulsgeber.

Um die Schwelle zum selbständigen Erzählen so niedrig wie möglich zu halten, knüpften sie zunächst an die Alltagserfahrungen der Kinder an. Im September 2006 waren dies die Erinnerungen an die Sommerferien. So lag es nahe, beim Geschichtenerfinden darauf zurückzugreifen. Die folgenden Beispiele aus einem Verlaufsprotokoll stammen aus einer zweiten Klasse. Die Klasse hatte bereits ein halbes Schuljahr an dem Projekt teilgenommen.

1.

Junge: Es war einmal ein Dorf. Da war ein Esel. Da bin ich drauf geritten.

2.

Mädchen: Es war einmal ein Flugzeug. Da bin ich drauf geflogen in die Türkei und da war ein Meer und da bin ich rein gesprungen.

Andere Kinder orientierten sich an diesen beiden Geschichten, und es folgte eine Reihe ähnlicher Erzählungen mit minimalen Variationen. Der märchenhafte Anfang hatte sich als Startsignal für eine Geschichte eingeprägt, aber die Phantasie bleibt - der Aufgabenstellung entsprechend - dem Alltagsgeschehen verhaftet. Dann erfolgte eine überraschende Wendung:

1.

Mädchen: Es war einmal, ich hab einen Mann gesehen. Der aß einen Igel.

Die Kinder lachten irritiert, fragten nach, ob dies tatsächlich geschehen sei, und das Mädchen zwinkerte mit den Augen und gab so zu verstehen, dass sie geflunkert habe.

Nun war die Phantasie der Kinder geweckt, und sie vermischten Fiktion und Realität:

1.

Junge: Es war einmal ein Hotel und da war ein Hai drin!

2.

Junge: Es war einmal in Italien. Da war ein Schwein. Er wollte mich auffressen.

Intuitiv hatte dieser Junge ein spannungsauslösendes Moment, einen Konflikt in seine Geschichte installiert. Auch das fand sofort Nachahmung:

1.

Mädchen: Es war einmal ein Meer. Da habe ich einen Hai gesehen. Er wollte mich fressen. Ich bin so schnell geschwommen. Dann bin ich dahin gegangen, wo meine Mutter war und da sind wir weggegangen. 
Die Geschichte liest sich wie die Minimalvariante einer Abenteuergeschichte und ist nach deren Grundprinzipien aufgebaut: Exposition (Person, Ort, Handlungssituation), Komplikation (Bedrohung und reaktives Handeln), Lösung. Es lässt sich vermuten, dass die Kinder diese simple Verlaufsform, nach der in der Regel die Volksmärchen gebaut sind, im Zuhören so verinnerlichen konnten, dass sie ihnen nun zum kreativen Gebrauch zur Verfügung stehen.

Diese Vermutung wird durch eine Vielzahl ähnlicher Beispiele erhärtet, so u.a. durch die vier Geschichten eines Mädchens, die sie innerhalb von 5 Monaten erzählte. Auch sie war im September 2006 bereits ein halbes Jahr am Projekt beteiligt:

1.

08.09.06: Es war einmal in Österreich. Da bin ich Auto gefahren. Zum Supermarkt.

2.

09.11.06: Es war einmal ein schöner Tag. Da waren die mit dem Schiff raus gegangen. Die waren Piraten, und die waren fröhlich. Da war ein Wasserfall, da sind die runter gefallen. Da waren Elfen und andere schöne Sachen. Die Piraten haben das nicht geglaubt. Das ist das Ende.

3.

18.12.06: Es war einmal ein Haus, und da spukte es drin, und als ich rein kam, da war so ein Baumstamm, da war eine Eule drin. Die wollte mich fressen. Da hab ich die mit dem Baumstamm erschlagen, dann hab ich das vergraben. Dann kam ein schöner Prinz heraus und den habe ich geheiratet.

4.

11.01.07.

: Ich habe noch eine Geschichte aus dem Kopf: Es waren einmal ein Mann und eine Frau, die waren ganz arm und wollten ein bisschen Geld haben. Der Mann fischte immer und die Frau putzt (aus: Fischer Palunko). Als die Frau mal frische Luft schnappen ging, kam ein Mann geritten. Der gab ihr eine Tüte und sagte: „Da könnt ihr euch was wünschen und es geht in Erfüllung (aus: Iwanuschka Dummkopf). Aber leise und immer in die Tüte rein." Da wünschten sie sich alles und wurden reich und lebten in einem Schloss. Und von dem Geld haben sie allen Armen gegeben und dann waren alle reich. Der Mann hat eines Tages einen Riesenfisch gefangen. Als er ihn aufschlitzte und öffnete, waren Goldstücke drin (aus: Der Fischer und sein Traum). Er sagt: „Wir nehmen die und machen daraus Geld." Das haben sie gemacht und allen anderen Geld gegeben, dass die auch was haben.

Bereits in der zweiten Geschichte nimmt dieses Mädchen ein fiktionales Sujet auf - die Piraterie -, das sich unvermittelt mit einem Märchenmotiv vermischt: die 
Elfen als Schatzbewahrer. Die Konstruktion ist noch ungelenkt und mutwillig, aber die Phantasie erprobt sich gleichsam schon an einer anderen Partitur. In der 4. Geschichte triumphiert die für das Märchen (beinahe) obligatorische Wunscherfüllung und überformt die Kalamität des Alltags auf wunderbare Weise. Die Frau, die putzt, und der Mann, der fischt, sind erstaunliche Legierungen aus Realität und Märchen, und durch die Wendung des Schicksals mit dem Auftauchen einer geheimnisvollen Helferfigur wird nicht nur ein individuelles Dilemma gelöst, sondern die Glückserfüllung erweitert sich auf ,alle Armen' - eine berührende Form kindlicher Utopie.

Als besonders ergiebig erwies sich ein Verfahren, bei dem die Erzählerinnen einen Anfang vorgaben, der märchennahe Phantasien auslöst und aus dem die Kinder gemeinsam mit der Erzählerin eine Geschichte entwickelten. Die Erzählerin griff nur dann ein, wenn die Geschichte durch ein Übermaß an Wiederholungen oder Nebenhandlungen an Spannung verlor oder wenn sich die Handlungsstränge heillos zu verwirren drohten. Im Wechsel von Lenkung durch die Erzählerin und freiem Phantasieren der Kinder entstanden komplexe Geschichten, die in Struktur, Motivik und Ikonografie aus dem reichen Schatz der erinnerten Märchen schöpften. Mit diesem ,Recycling-Verfahren' wuchs das Vertrauen der Kinder in ihre eigene Erzählkompetenz und die Freude am inspirierenden Miteinander beim Erfinden neuer Geschichten. Ein Beispiel für eine auf diese Weise entstandene Erzählung:

Vorgabe der Erzählerin: „Es war einmal ein Junge, der ging in den Wald. Dort begegnete ihm..."

Die Kinder ergänzen die Geschichte in folgender Weise: „Ein Junge ging in den Wald und wollte Holz fällen. Da begegnete er einem kleinen Zwerg. Der Zwerg sagte: „Das ist mein Wald. Du darfst hier nicht Holz fällen. Nur wenn du mir, dem Zauberzwerg, ein Geschenk mitbringst." Da lief der Junge nach Hause und holte eine goldene Kette, denn er war reich. Da gab ihm der Zwerg ein Goldstück. Von nun an durfte der Junge in dem Wald Holz fällen. Eines Tages traf er wieder den Zwerg, und dieser stand neben einem Stein. „Dies ist ein Zauberstein“, sagte der Zwerg, „und du darfst ihn nicht berühren, sonst wird der böse Zauber daraus befreit." Denn in diesem Stein war ein böser Zwerg verschlossen. Aber der Junge war so neugierig, und eines Tages ging er zu dem Stein, lief dreimal darum, und dann öffnete sich dieser in der Mitte, und heraus zischte ein kleines rotes Männchen und rief: „Haha, jetzt bin ich befreit!“ Dann flog es in die Luft. Es richtete überall in der Welt Unheil an und großes Chaos, und Dunkelheit kam über die Welt. Da gab der gute Zwerg dem Jungen ein fliegendes Pferd, das sich in ein Eisenpferd verwandeln konnte, einen Zaubermantel, der unsichtbar machte, und eine Zaubermütze, die, einmal aufgesetzt, nicht mehr zu entfernen war. Damit ausgestattet, ging der Junge auf die Suche nach dem bösen Zwerg. Dieser lebte in einem kleinen Wald. Der Junge schlich sich in seinem unsichtbaren Mantel an, als der böse Zwerg schlief, und setzte ihm dann schnell die Mütze auf.

Da die Erzählerinnen beobachteten, in wie starkem Maße sich die Kinder beim 
Erzählen gegenseitig inspirierten, gingen sie immer öfter dazu über, die Klassen in Kleingruppen von 3 bis 4 Kinder zu teilen und diese gemeinsam Geschichten erfinden zu lassen.

Da ein Großteil der europäischen Volksmärchen nach dem Schema des klassischen Dramas aufgebaut sind (Exposition, auslösendes Moment, aufsteigende Handlung/Komplikation, Klimax/Höhepunkt, retardierendes Moment, Lösung $)^{2}$, nutzten die Erzählerinnen diese strukturelle Gebundenheit, um die Komplexität eines Märchens für die Kinder fassbar zu machen und den Erzählvorgang zu strukturieren. Im Anschluss an ein erzähltes Märchen rekapitulierten sie nochmals gemeinsam mit den Kindern den Handlungsverlauf und unterteilten ihn in fünf oder drei ,Akte'. Durch diese Segmentierung wurden die Geschichten überschaubarer und damit auch besser memorierbar. Jedes Kind wählte im Anschluss an diese Repetition einen ,Akt', den es besonders interessant fand, aus und malte dazu ein Bild. Anschließend wurden die Bilder wie in einem Storyboard zusammengesetzt, und die Kinder konnten das Märchen bildgestützt nacherzählen.

Weiterhin setzten die Erzählerinnen bildgestützte Verfahren ein, welche die Phantasie der Kinder in besonderer Weise anregten. Sie legten den Kindern Märchenkarten vor, aus denen sie jeweils drei ziehen konnten. Auf den Abbildungen fanden sich Personen, Orte, Tiere oder Gegenstände. Mit ihrer Hilfe sollten die Kinder ein Märchen erfinden.

Fiel es den Kindern zunächst schwer, die drei Motive zu einer schlüssigen Geschichte zu verknüpfen, so lernten sie doch verblüffend schnell, fantasievolle und folgerichtige Brücken von einer Karte zur nächsten zu schlagen.

Mit viel Sympathie beantworteten die Kinder die Aufforderung der Erzählerinnen, aus Bananenkisten ein japanisches Kamishibai ${ }^{3}$ zu bauen und die Bilder für dieses Erzähltheater selbst zu malen bzw. mit Collagetechniken anzufertigen. Die Bilder stützten die Erinnerung und halfen den Kindern, die Geschichte als Ganzes zu rekapitulieren. Der Zuwachs an Erzählkompetenz, vor allem aber auch an Selbstvertrauen war bei den Präsentationen vor der Klasse deutlich zu spüren.

Insgesamt wurden solch bildgestützte Verfahren sparsam eingesetzt, um die Vorstellungswelt der Kinder nicht - wie gewohnt - durch visuelle Vorgaben zu dominieren, sondern eigenständige Bilder im Kopf entwickeln zu lassen

In einem anderen Verfahren sollten die Kinder Varianten eines erzählten Märchens erfinden. So wurden sie z.B. aufgefordert, einen männlichen Protagonisten durch eine weibliche Hauptfigur zu ersetzen. In diesem Verfahren ist das Gerüst für einen möglichen Verlauf der Geschichte vorgegeben, und auf dessen Grundlage können die Kinder frei fantasieren, ohne zu fürchten, den Faden zu verlieren.

Die Erzählerinnen entzündeten die narrative Phantasie der Kinder auch, indem sie besonders prägnante und/oder ästhetisch anspruchsvolle Objekte zur Verfügung stellten: Goldene Schlüssel, ein Spinnrad, eine silberne Kanne

\footnotetext{
${ }^{2}$ Vgl. dazu ausführlich Wardetzky 2007: 46 ff.

${ }^{3}$ Siehe dazu: Rost / Beasecke 2007: 62 ff.
} 


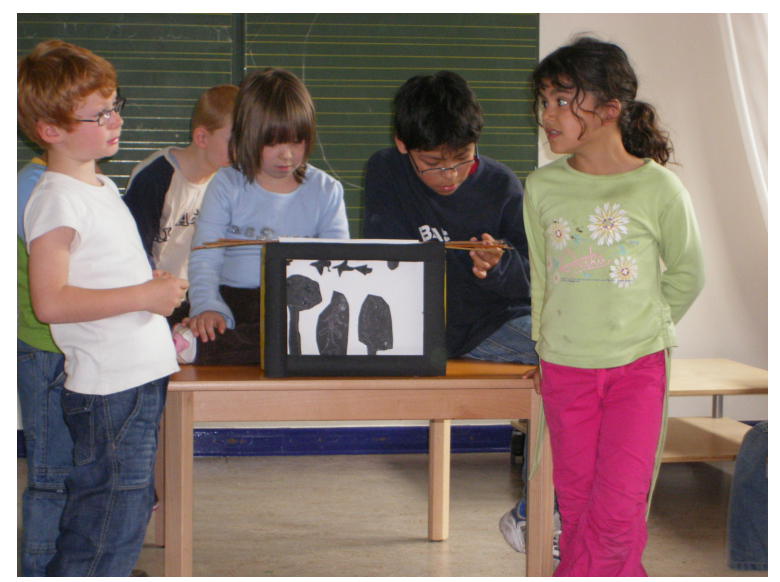

Abbildung 4: Kinder erzählen mit Kamishibai

usw. Solche Gegenstände tragen die Spuren gelebten Lebens in sich. Sie sind Geschichtentresore, die aufgeschlossen und ,zum Reden gebracht' werden wollen.

Auch der Aufforderung der Erzählerinnen, besonders kostbare Gegenstände von zuhause mitzubringen, die in einem (losen) Zusammenhang mit einer erzählten Geschichte stehen könnten, kamen die Kinder gern nach. Sie waren stolz, ihre ,Schätze' zu zeigen, erzählten Geschichten dazu und verwendeten sie dann für die Rekapitulation ausgewählter Märchen.

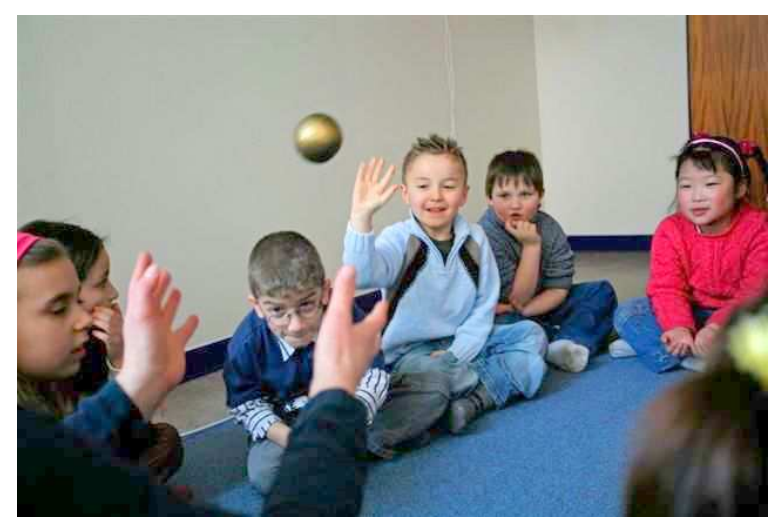

Abbildung 5: Erzählen mit Objekten

\section{Zusammenfassung}

Am Ende seien die wesentlichen Besonderheiten des hier vorgestellten Projekts zusammengefasst:

Bezogen auf die Schule schließt dieses Projekt die Kluft im Bereich der Bildungschancen: Künstlerisches Erzählen erreicht alle Kinder. Es ist d i e demokratische Form der Literaturvermittlung. Darüber hinaus kann das Projekt 
an jeder Schule umgesetzt werden: Es bedarf keiner technischen Ausstattung und ist in diesem Sinne kostenunaufwändig und flexibel.

Bezogen auf die Schüler erweist sich das Projekt als ein ideales Verfahren, Kinder an die deutsche Sprache heranzuführen. Davon profitieren insbesondere Kinder mit Migrationshintergrund, in deren Elternhäusern nicht Deutsch gesprochen wird, sowie Kinder aus sozial schwachen Milieus und Kinder mit erheblichen Lernschwierigkeiten. Die Sprach- und Erzählkompetenz der Kinder erweitert sich in einem Lernprozess, in dem sich rationales und emotionales Verstehen auf fruchtbare Weise ergänzen. In besonderer Weise wird die Lust am Gebrauch einer poetischen Sprache geweckt.

Darüber hinaus erweist sich das Erzählen von Märchen als Grundschule literarischer Bildung. Über das Erzählen von Märchen werden die Kinder an Zeugnisse der Weltliteratur herangeführt und ihre Neugier auf die Herkunftskultur ihrer Eltern sowie auf andere Kulturen wird angeregt - ein Weg zur gegenseitigen Verständigung und Akzeptanz. Damit werden Integrationsprozesse gefördert, die die Wahrnehmung der Besonderheiten verschiedener Kulturen einschließen.

Die Konzentrationsfähigkeit der Kinder, d.h. ihre unabgelenkte Aufmerksamkeit auf die Verlebendigung des gesprochenen Wortes, wird in erheblichem Maße gestärkt. Das Gleiche gilt für die Entwicklung der Phantasie, d.h. für die Fähigkeit, fiktive Welten durch eigene, imaginierte Bilder zu erschaffen.

Über das Märchen werden für die Kinder existenzielle Probleme ihres Alltags kommunizierbar und die Fähigkeit wird entwickelt, die Welt anders zu denken als sie ist. Damit wird das Erzählen zur Schule des alternativen Denkens.

Die Erfolge des Projektes sind zum einen an die Langfristigkeit und Intensität der Begegnung mit dem mündlich vermittelten Märchen gebunden, zum anderen an die Professionalität der Erzählerinnen. Beide Voraussetzungen sind unabdingbar, will man ähnliche Erfolge bei der Übertragung des Projektes auf andere Schulen erreichen.

Das Projekt lädt dazu ein, auf die Wirkungsmacht des Erzählens in der Schule aufmerksam zu machen, insbesondere bei der Vermittlung des Deutschen als Zweitsprache. Lehrer, die sich für die Wiederentdeckung des Erzählens in der Schule stark machen, können durch die Rückbesinnung auf alte literarische und pädagogische Traditionen nachhaltige Veränderungen im Bereich der Sprachförderung anstoßen.

\section{Bibliographie}

Wardetzky, Kristin (2007): Projekt Erzählen. Hohengehren

Rost, Hedwig / Baesecke, Jörg (2007): Höher als der Himmel, tiefer als das Meer. Ein Geschichtenwerkbuch. Frankfurt / Main 


\title{
Appendix:
}

\author{
Beispiele aus der Dokumentation: \\ Nacherzählungen von Kindern aus Interviews
}

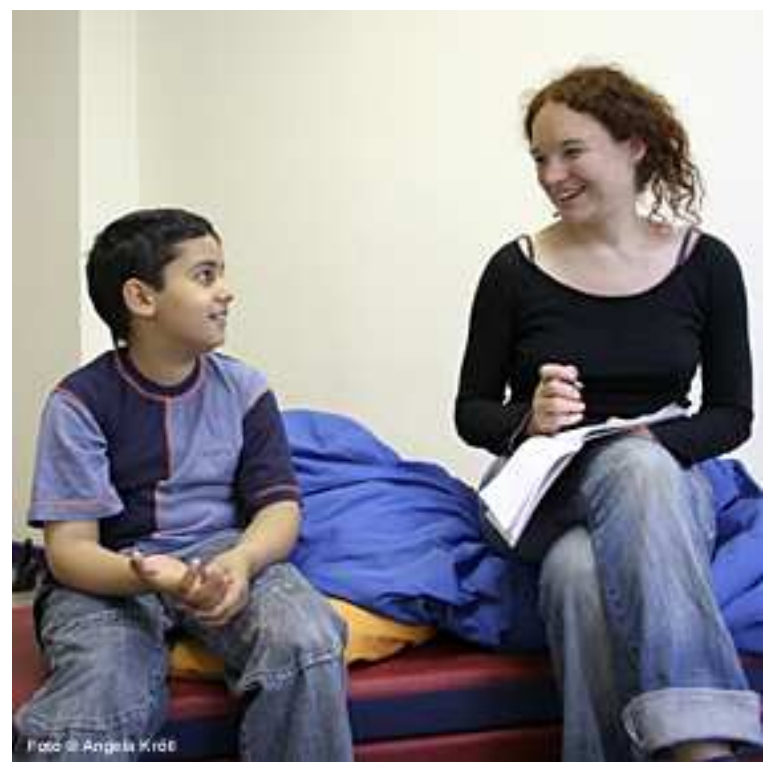

Abbildung 6: Interview

Im zweiten Schuljahr des Projektes führte Christiane Weigel (CW) mit den Kindern, die jeweils ein halbes oder ein Jahr am Projekt beteiligt waren, persönliche Interviews, in denen die gehörten Geschichten nacherzählt bzw. kommentiert oder eigene Geschichten erfunden werden sollten. Die Interviews geben zum einen Aufschluss darüber, woran sich die Kinder erinnern konnten, wie sie Verständnislücken schlossen oder Gehörtes in ihrem Sinne umdeuteten. Zum anderen dokumentieren diese Erzählungen die Entwicklung der Erzählkompetenz der Kinder. Dabei läßt sich überprüfen, inwieweit die Sprache der Erzählerinnen den Sprachgebrauch und die Ausdrucksfähigkeit der Kinder beeinflusst hat.

Die Gesprächssituation machte deutlich, wie stark die Kinder die ungeteilte Zuwendung und Aufmerksamkeit der Interviewerin genossen. Die Erfahrung, dass ein Erwachsener ihnen geduldig und ermunternd zuhört, ihre Freude am Suchen bzw. Finden von Wörtern und Wendungen teilt, gehört ganz offensichtlich zu den seltenen glücklichen Augenblicken ihres Alltags. Erwachsene als Zuhörer - dies scheint ebenso unabdingbar für die Entwicklung von Erzählkompetenz wie das ,Füttern' mit Geschichten.

Waren die im Interview protokollierten Nacherzählungen der Kinder in den ersten Monaten häufig fragmentarisch und lückenhaft, so erhielten die Geschichten immer stärker ihre ursprüngliche Struktur und logische Dramatik. Auffällig war, dass die Kinder in den ersten Monaten häufig den Schluss eines Märchens nicht mehr recht erinnerten, weil sie, offensichtlich mit ihrer 
Konzentration am Ende, nicht bis zum Schluss zugehört hatten. Im zweiten Halbjahr konnten die Kinder überwiegend die gesamte Geschichte nacherzählen. Dies mag zum einen mit der gewachsenen Konzentrationsfähigkeit zusammenhängen. Zum anderen dürfte eine beträchtliche Rolle spielen, dass die Kinder im Laufe der Zeit mehr Kenntnis über die Struktur eines Märchens gewonnen hatten und sich somit besser innerhalb einer Geschichte orientieren und diese anschließend strukturierter und vollständiger nacherzählen konnten.

A. (Türkei) und S. (Jugoslawien) erzählen das afrikanische Märchen „Mbango" Für A., ein stilles Mädchen, das auch im Unterricht wenig spricht, ist das Erzählen mit großer Anstrengung verbunden. Dennoch verweigert sie sich nicht, sondern will sich mitteilen und erzählen - ein Bedürfnis, das man bei ihr im Unterricht sehr selten beobachten kann. Sie erinnert die Sukzession der Bilder (die Insel, das Fressen und den Schluckauf der Schildkröte, das Seil usw.), Aktionen, die sie auf bekannte Situationen ihres Alltags übertragen kann (das Ziehen am Seil, das Kommando ,Achtung, fertig, los'), vor allem aber den freudvollen Schluss (die ungleichen Tiere werden Freunde und tanzen). D.h., sie hat bis zum Ende zugehört, der eigentliche Witz der Geschichte (die Schwachen überlisten die Starken/Großen) ist ihr nur ahnungsweise zugänglich (,Kann ich nicht so gut erinnern').

Im Unterschied zu A. steht eben diese List für S. im Mittelpunkt seiner Erzählung, und er kostet das trickreiche Handeln der Schildkröte mit sichtlichem Behagen aus.

A.: Schildkröte ist zu der Insel hin gegangen. Aber dann die Insel war ganz groß. Dann hat auch noch die Schildkröte Blätter gegessen. Dann ist irgendwas Großes gekommen. Ich glaub, das war das Nilpferd. Hatte die Schluckauf, die Schildkröte. Die Schildkröte hat dem Nilpferd gesagt, dass die einen Kampf machen sollen. Meinte das Nilpferd: „Okay!“ Ist die Schildkröte, hat das zum Elefanten gesagt. Hat die, glaub ich, die Schildkröte gesagt, dass das Nilpferd stärker ist als das Elefant. Kann ich nicht so gut erinnern. Hat die Schildkröte auch gesagt, dass Kampf machen sollten. Hat der Elefant auch gesagt: „Okay!“ Schildkröte ist zum Nilpferd gegangen und gesagt, dass es das Seil halten soll. Hat er auch. Dann ist die Schildkröte noch zum Elefanten gegangen. Hat der auch das Seil genommen. Dann ist er zum Baum gegangen in die Mitte. Hat die Schildkröte gesagt: „Auf die Plätze, fertig, los!“ Haben die los gezogen. Hat der Elefant ein bisschen gezogen, das Nilpferd ein bisschen. Später haben sie beide das Seil los gelassen. Schildkröte zum Nilpferd gegangen. Hat der gesagt, dass die Schildkröte stark ist, klein und stark. Sind die Freunde. Dann bei Elefanten das Gleiche. Dann ist die Schildkröte auch Freundin von Elefant. Dann haben sie getanzt.

S.: Die Schildkröte war im Wasser. Ist zu die Insel gegangen. Hat die einen Schritt gemacht. Dann ist das zu einen Nilpferd gewachsen. War die Insel so groß. Dann hatte die Schildkröte ein Blatt genommen und hat das gegessen. Dann kam ein Geräusch, und dann hatte sie Angst. Da war 
ein Nilpferd, und da war noch ein Elefant. Dann waren die stark. Ist die genau zu das Nilpferd gegangen und wollte mit die kämpfen, wenn die Sonne ganz hoch steht. Dann ist die Schildkröte zu den Elefant gegangen: „Ich will mit dir kämpfen“. Elefant sagt: „Okay. Wann?“ Schildkröte: „Um Zwölf, wenn die Sonne ganz hoch steht." Die Schildkröte war klug, ist die genau in die Mitte gegangen und hat gesagt: „Wenn ich los sage, könnt ihr anfangen.“ Und dann hat die „Auf die Plätze, fertig los!“ gesagt. Das Nilpferd und der Elefant haben am Seil gezogen. Und die Schildkröte war in der Mitte. Die Schildkröte hat so geguckt, dann konnten die nicht mehr. „Ja Schildkröte, was bist denn du für ein starkes Tier?" War es ein ,Sie' oder ein ,Er'?

C.W.: Ich glaube, eine, sie'.

S.: Hat die Schildkröte gesagt: „Ich bin ja stark.“ Und das Nilpferd: „Willst du meine Freundin sein?“ Schildkröte: „Ja, ich will.“ Ist die Schildkröte zum Elefanten gegangen. Der Elefant hat sich wieder aus dem Baum so raus geholt mit den Rüssel. Elefant: „Schildkröte, warum bist du denn so stark?" Schildkröte: „Ja, ich bin eben so stark.“ „Willst du meine Freundin sein?" Schildkröte: „Ja, ich will.“ Dann haben die den Tanz gemacht. Dann wusste nicht das Nilpferd, was es machen sollte. Ist das auch hin gegangen. Dann haben die alle getanzt.

D. (Deutschland) und S. (Serbien) erzählen das Märchen „Die drei Schwäne“ Auch D. kannte am Anfang des Projektes keine Märchen und war im freien Erzählen ebenso unerfahren wie alle ihre Klassenkameraden. Die Gewandtheit, mit der sie dieses komplexe, in sich verschachtelte Schwanenjungfraumärchen rekapituliert, wie sie konsequent den Handlungsfaden verfolgt und die unterschiedlichsten Motive in ihn hineinwebt, wie sie das (mythische) Alter der hilfreichen Zauberin durch die fünfmalige Wiederholung des Attributs ,alt' deutlich macht, wie sie aus dem Feuervogel Phönix die Kontamination Feuerphönix kreiert, wie sie das Vergehen der Zeit durch die wiederkehrende Wiederholung der gleichen formelhaften Wendung verdeutlicht - das ist für Zweitklässler durchaus ungewöhnlich.

D.: Der Opa, der hatte drei junge... Wie heißt das noch mal?

C.W.: Meinst du Söhne?

D.: Ja. Drei junge Söhne. Und der Opa hatte draußen bei seinem Garten geschlafen. Und da waren so Schwäne, Prinzessin und Dienerinnen. Der Opa hat es nicht gesehen, weil er eingeschlafen war. Dann hat der große Sohn es probiert und ist auch geschlafen. Sagt der Kleine: „Kann ich das jetzt mal versuchen?“ „Nein. Du bist zu jung.“ Dann hat er es doch gemacht und er ist nicht eingeschlafen. Er hat Lieder gesungen und sich selber Geschichten erzählt. Dann sind die Schwäne gekommen, also die Königstochter und die zwei Dienerinnen. Haben die ihre Federn abgenommen und rumgetanzt und in Kreis gedreht. Dann hat er die Federn genommen. Dann haben die die Federn gesucht. Dann kam der kleine Junge und hat gesagt: „Was sucht ihr denn?“ „Wir suchen unsere 
Federn.“ „Ich weiß, wo sie ist. Aber erst muss die Schönste meine Frau werden." "Okay." Dann sind die geflogen. Vorher haben die noch gesagt, dass abends die Hochzeit ist. Dann haben die alles vorbereitet, und dann haben sie geheiratet. Dann ist sie wieder weggeflogen, und er wartet, dass sie wiederkommt. 1 Tag, 2 Tage, 1 Jahr, 1 Monat. Dann hat er sie gesucht. Essen und Trinken eingepackt und gelaufen in den Wald. Da hat er solche Trolle gesehen im Wald, ganz laut. „Warum streitet ihr euch über ein Paar Schuhe?“ „Unser Papa ist tot und diese Schuhe sind etwas ganz besonderes. Man kann damit $1 \mathrm{~km}$ oder...?

C.W.: Hundert Meilen in einer Minute.

D.

: „Warum gebt ihr mir sie nicht?“ „Okay!“ sagen die dummen Trolle. Die sind doof. (Sie amüsiert sich über deren Dummheit.) Dann ist er gelaufen und kam wieder in den Wald und hörte Trolle streiten. „Warum streitet ihr wegen so nen alten Mantel?" „Unser Papa ist gestorben und der ist ganz besonders. Wenn man den anzieht, wird man unsichtbar.“ „Warum gebt ihr mir nicht den Mantel? Dann müsst ihr euch nicht mehr streiten.“ Haben die ihm den Mantel gegeben. Dann ist er $100 \mathrm{~km}$ gelaufen und hat dann wieder die Geräusche gehört. So Brüllen von Trollen. War da so ein Schwert. Wenn man jemand die Spitze zeigt, ist er tot. Wenn man jemand mit den Griff berührt, ist er wieder lebendig. „Warum gebt ihr mir nicht das Schwert?" Dann hat er das Schwert auch bekommen. Dann hat er eine alte, alte, alte, alte, alte Frau getroffen und gefragt: „Können Sie mir sagen, wo das Schloss ist östlich der Sonne und nördlich der Erde?“ „Nein, aber vielleicht meine Tiere." Sie hat 3x geklatscht, und dann kamen alle Tiere. „Wisst ihr, wo das Schloss ist, östlich der Sonne und nördlich der Erde?“ „Nein.“ Dann hat er wieder eine alte, alte, alte, alte, alte Frau getroffen. „Haben Sie ein Schloss gesehen östlich der Sonne und nördlich der Erde?" „Nein, aber ich ruf mal meine Meerestiere." Hat sie getan, $3 \mathrm{x}$ geklatscht. Hat sie gefragt. Haben sie nein gesagt, sind sie wieder zurück gegangen. Ist er weiter gelaufen. Ist er wieder an eine alte, alte, alte, alte, alte Frau angekommen. „Haben Sie ein Schloss gesehen östlich der Sonne und nördlich der Erde?" „Nein, aber ich ruf mal meine ganzen Vögel, Spätze und so." Hat sie wieder 3 x geklatscht, alle sind gekommen außer... Wie heißt der noch mal?

C.W.: Phönix, der Feuervogel

D.: außer Feuerphönix. „Einer fehlt! Der Feuerphönix!“Kommt er so müde und so kaputt. „Hast du ein Schloss gesehen östlich der Sonne und nördlich der Erde?“ „Ja, da war ich gerade.“ „Dann kannst du gleich den Jungen dahin fliegen!" Er mags nicht oder er mag (sie ersetzt damit die Formulierung ,ob er will oder nicht'). Ist er auf seinen Rücken gegangen, und sind sie geflogen. War so eine Wolke, und dann waren sie da. Hat er geklopft. Mit einem langen Bart, weil er ein, zwei Tage, ein Monat, ein Jahr hin und her gegangen ist. „Wer ist da?“ hat die Dienerin gefragt. „Ich bins, der Mann 
von der Königstochter.“ Hat sie gesagt: „Nein, du bist das nicht.“ Hat er erstmal den Apfel raus geholt. „Das sag ich der anderen Dienerin.“ (Sie spielt jetzt die Figuren.) Die hat gesagt: „Das glaub ich nicht.“ „Komm mal mit!“Sie denkt es auch nicht. Aber dann holt er den linken Apfel. „Ach du bist der! Den linken Apfel erkenn ich.“ Gehen sie zur Königstochter: „Dein Mann ist da." Glaubt sie auch nicht. Hat er den Ring gezeigt. „Richtig, du bist das! Aber wir können dich nicht rein lassen. Die Trolle sind da.“ „Das macht nichts. Ich kann was machen. "Dann hat er sich unsichtbar gemacht und hat alle mit dem Schwert getötet. Dann konnten sie eigentlich gehen.

C.W.: Da war noch jemand tot.

D.

: Ja, die Königstochter sagt dann: „Aber meine Eltern sind gestorben.“ „Ich kann was dagegen tun." Sind sie zum Grab gegangen, haben das erstmal aufgemacht. Hat er mit dem Griff berührt. Dann die Augen waren auf (sie sagt es mit großem Staunen, scheint es vor sich zu sehen) und sie lebten wieder. Haben sie dann dem den Bart abrasiert und haben den zum schönsten Jungen gemacht. War da dann noch was?

C.W.: Die haben noch gefeiert.

D.

: Dann haben sie Hochzeit gefeiert und Saft und Cola und Bier getrunken. Ja, manche Leute trinken das. Und dann gab es Kirschkuchen, Himbeerkuchen, Sahnekuchen. Das ist mein Lieblingskuchen.

C.W.: Hat dir die Geschichte gut gefallen?

D.: Ja sehr.

Das gleiche Märchen erzählt S, ein Junge nicht-deutscher Herkunftssprache, ebenfalls 2. Klasse. Ganz offensichtlich beeindruckt von den Zaubergegenständen, beginnt er seine Nacherzählung mit deren Aufzählung. Um ihre Wirkung zu verdeutlichen, ergänzt er das von der Erzählerin Gehörte - mit den Schuhen ist der Held der Geschichte fast eine ganze Runde um die Erde gelaufen. Auch er weiß das Märchen in allen Details zu rekapitulieren. Die von ihm gewählten Substitutionen machen deutlich, wie sich Verstehensvorgänge konstituieren: Anfangs ersetzt er ,Trolle' - ein für ihn unbekannter Begriff, den die Erzählerin nicht eingeführt hatte - sinnentsprechend durch Riesen, später nimmt er die eigene Sinndeutung wieder zurück und verwendet den von der Erzählerin verwendeten Begriff. Die hilfreiche Alte macht er sinnentsprechend zur Oma, den Protagonisten zum ,jungen Burschen' - eine Formulierung, die er aus den Erzählstunden kennt. Aus ,Jemand hat mich so nett gegrüßt' macht er ,angegrüßt' - offensichtlich in dem Bestreben, die Besonderheit des zeremoniellen Grüßen kenntlich zu machen. Den ungekämmten Bart beschreibt er als ,zerfetzt' - eine stimmige Übertragung, um das veränderte Aussehen des Helden zu verdeutlichen.

S.: Da war ein Schwert. Wenn man jemanden trifft, ist er tot. Und wenn man am Griff jemanden berührt, lebt man wieder. Dann mit dem unsichtbaren 
Mantel. Und die Stiefel, die ganz schnell rennen können.

C.W.: Und weißt du noch, wie die Geschichte angefangen hat?

S.: Da waren drei Söhne. Der Vater ist dann auf die Wiese gegangen und hat gesehen, dass die Füße, da Fußabdrücke sind. Hat der zu dem Ältesten gesagt, er soll übernachten. Hat er sich ein gemütliches Plätzchen gesucht und Essen und Trinken ausgepackt, und dann ist der eingeschlafen. Der Mittlerste hat auch übernachtet. Der ist auch eingeschlafen. Dann hat der Kleinste gesagt, er will auch da übernachten. Hat der Papa gesagt: „Du kannst doch nicht. Du bist zu klein!“ Dann ist der raus gegangen und hat gesungen und sich Geschichten erzählt, also allein. Dann sind drei Schwäne gekommen, haben ihre Federn abgelegt, und dann war da eine, die so wunderschön war. Haben die getanzt und sich umgedreht. Dann hat der Kleine ihre Federn weggenommen. Dann als die Sonne kam, haben die ihre Federn gesucht. Ist der Kleine gekommen und hat gesagt: „Wer seid ihr?“ „Wir kommen nördlich von der Sonne und östlich von der Erde.“ Haben die gesagt: „Gib uns unsre Federn zurück. Wenn die Sonne kommt, dann sterben wir." Hat er zu der Prinzessin gesagt (die anderen waren nur ihre Dienerinnen): Er soll sie heiraten. Dann hat er ihre Federn gegeben und sie sind geflogen. Hat er alles dem Vater erzählt und dass die morgen kommen und ein Fest feiern, also Hochzeit. Haben die dann Hochzeit gefeiert und die Dienerinnen haben ihm 2 Äpfel gegeben und die Prinzessin ein Ring. Dann sind die weggeflogen. Er hat gewartet. Ein Tag ist vergangen und ein Jahr. Hat er sich Sorgen gemacht und hat die gesucht. Ist er zu zwei Riesen gekommen, die haben sich gestritten wegen zwei Schuhen. Hat er gesagt: „Wieso streitet ihr euch wegen zwei Schuhe?" „Unser Vater ist gestorben und er hat uns nur diese zwei Schuhe gegeben." Hat er gesagt: „Gebt die Schuhe doch mir, dann müsst ihr nicht streiten." Die waren ja dumm und haben ihm die gegeben. Dann ist er ganz schnell gelaufen mit den Schuhen, fast eine Runde um die Erde. Dann haben sich wieder zwei Riesen gestritten nur wegen einen Mantel. Sagt er: „Wieso streitet ihr euch denn nur wegen einen Mantel?" „Unser Vater ist gestorben, und er hat uns nur diesen Mantel gegeben und jetzt wissen wir nicht, sollen wir den zerschneiden.“ „Warum gebt ihr nicht mir den Mantel? Dann müsst ihr nicht streiten.“ Haben die ihm den Mantel gegeben. Das war ein unsichtbarer Mantel. Dann ist er wieder gekommen, da haben sich zwei Riesen gestritten nur wegen ein Schwert. „Wieso streitet ihr euch denn über dieses Schwert? Es ist doch alt und zerfällt.“ „Unser Vater ist gestorben und hat uns nur dieses Schwert gegeben.“ „Dann gebt das Schwert doch mir. Dann müsst ihr euch nicht mehr streiten." Das war ein Schwert: Wenn man jemanden mit der Spitze berührt, dann stirbt er und wenn man jemanden mit dem Griff berührt, dann lebt er. Dann ist er zu einem Wald gekommen. Da war eine alte, alte, alte Oma. Sie hat. . Dann hat er gesagt: „Hallo!“ Hat die Oma gesagt: „Jemand hat mich so nett angegrüßt. Ich lebe schon viele, viele Jahre, doch nie hat mich jemand so freundlich gegrüßt." Dann 
so: „Dann wächst etwas und dann wieder nicht.“ (Er ersetzt mit dieser Formulierung die Passage der Erzählerin ,Ich sah Eichenwälder wachsen und vergehen'.) Dann hat er gesagt, ob die jemanden kennt, wo die nördlich von der Sonne und östlich von der Erde. Hat sie gesagt: „Nein. Aber die Tiere wissen es vielleicht." Die konnte mit die Tiere reden. Hat die $3 \mathrm{x}$ in die Hände geklatscht, und dann sind die sofort gekommen. Hat sie gefragt: Wo ist die nördliche Sonne und die östliche Erde?" Aber die wussten das nicht. Dann ist er zu einem Meer gekommen. Da war eine alte, alte, alte Oma. „Sagt er: „Hallo!“ „Jemand hat mich so nett angegrüßt! Das Meer trocknet aus und dann fließt es wieder." Sagt er: „Weißt du, wo die nördliche Sonne und die östliche Erde ist?“ „Nein, aber die Fische wissen das vielleicht." Die konnte mit die Fische reden. Hat sie $3 \mathrm{x}$ geklatscht, und dann sind die Fische gekommen. Hat er gefragt: „Wisst ihr, wo die nördliche Sonne und die östliche Erde ist?" Aber die wussten das auch nicht. Er ist dann zu ein altes Haus wieder gekommen. War wieder eine alte Oma. Hat er wieder gesagt: „Hallo!“ Dann hat er wieder gesagt: Weißt du, wo die östliche Sonne und die nördliche Erde ist?" „Ich weiß es nicht.“ Dann hat sie 3 x geklatscht. Sie konnte mit die Vögel reden. Dann sind alle Vögel gekommen, aber jemand hat gefehlt. Das war der Feuervogel. Er kam dann. Er war müde. Er war lahm. Fragen die anderen: „Wieso brauchst du denn so lange?“ „Weil ich war bei der östlichen Sonne und nördlichen Erde.“ Sagt die Frau: „Gut, dann kannst du den jungen Burschen zu diese Sonne bringen." Hat er gemacht. Da war eine große Wolke, dann waren die da. Haben angeklopft an die Tür. Ist die Dienerin gekommen, der er den Apfel gegeben hatte. Er war sehr, sehr alt. Er hatte einen Bart und war zerfetzt. „Wer bist du?" hat sie gesagt. Hat er ihr den Apfel gezeigt. Da wusste die, dass er es ist. Dann ist die andere Dienerin gekommen. „Wer bist du?" Hat er ihr den anderen Apfel gezeigt. Dann ist die Prinzessin gekommen. Die wusste auch nicht, wer er ist. Hat er ihr den goldenen Ring gezeigt. Dann konnte er rein. Er konnte aber nicht hier bleiben, weil die Trolle kommen. Da hat er sich unsichtbar gemacht. Dann ist ein Troll gekommen und der nächste und der nächste. Dann waren die alle tot. Dann hat die Prinzessin gesagt: „Aber ein was ist noch. Meine Eltern sind tot." Dann hat er mit den Griff sie berührt, und dann waren die wieder gelebt. Dann haben die gefeiert und eine Hochzeit gemacht.

C.W.: Und was hat dir an der Geschichte am besten gefallen?

S.: Der unsichtbare Mantel.

C.W.: Und was ist dein Lieblingsmärchen?

S.: Das von heute. 